PESQUIMAT, Revista de la F.C.M. de la

Universidad Nacional Mayor de San Marcos

Vol. XV N², pp. 70-87, Lima - Perú, Marzo 2013

\title{
EXISTENCIA LOCAL Y NO EXISTENCIA GLOBAL PARA UN SISTEMA DE ECUACIONES DE ONDA NO LINEAL CON OPERADOR p-LAPLACIANO
}

\author{
Teófanes Quispe Méndez ${ }^{1}$, Yolanda Santiago Ayala ${ }^{2}$, Félix Pariona Vilca ${ }^{3}$
}

Resumen: Consideramos un problema mixto para un sistema de ecuaciones de onda no lineal con operador p-Laplaciano y con término disipativo fuerte. Probamos la existencia local de soluciones por el método de Galerkin y la explosión de soluciones por el método de la energía. Damos algunas estimativas para el tiempo de vida de las soluciones.

Palabras Claves: Solución local, Método de Galerkin, Explosión de soluciones, Sistema de ecuaciones de onda con Operador p-Laplaciano.

\section{LOCAL EXISTENCE AND GLOBAL NONEXISTENCE FOR A SYSTEM OF NONLINEAR WAVE EQUATIONS WITH p-LAPLACIAN OPERATOR.}

\begin{abstract}
We consider a mixed problem for a system of nonlinear wave equations with p-Laplacian operator and with dissipative strong term. We prove the local existence of solutions by Galerkin method and blow-up of solutions by the energy method. We give some estimates for the life span of solutions.
\end{abstract}

Key Words: Local solution, Galerkin method, Blow-up of solutions, System the wave equations with p-Laplacian operator.

\section{Introducción}

En este artículo consideramos el problema de valores iniciales y de frontera para el si-guiente sistema de ecuaciones de onda no lineal con operador p-Laplaciano:

$$
\begin{aligned}
& \left.u^{\prime \prime}-\Delta_{p} u-\Delta u^{\prime}=f_{1}(u, v) \text { en } \Omega \times\right] 0, \infty[, \\
& \left.v^{\prime \prime}-\Delta_{p} v-\Delta v^{\prime}=f_{2}(u, v) \text { en } \Omega \times\right] 0, \infty[,
\end{aligned}
$$

con condiciones iníciales

$$
\begin{aligned}
& u(x, 0)=u_{0}(x), u^{\prime}(x, 0)=u_{1}(x), \text { en } \Omega, \\
& v(x, 0)=v_{0}(x), v^{\prime}(x, 0)=v_{1}(x), \text { en } \Omega,
\end{aligned}
$$

y condiciones de frontera

$$
\begin{aligned}
& u(x, t)=0, \text { en } \partial \Omega \times] 0, \infty[, \\
& v(x, t)=0, \quad \text { en } \partial \Omega \times] 0, \infty[,
\end{aligned}
$$

donde $\Omega$ es un conjunto abierto y acotado de $\mathbb{R}^{n}$ con frontera suficientemente regular $\partial \Omega, \Delta$ es el operador Laplaciano, $\Delta_{p}$ es el operador p-Laplaciano definido por

$$
\Delta_{p} w:=\operatorname{div}\left(|\nabla w|^{p-2} \nabla w\right),
$$

\footnotetext{
${ }^{1}$ UNMSM, Facultad de Ciencias Matemáticas, E-mail: tquispem@gmail.com

${ }^{2}$ UNMSM, Facultad de Ciencias Matemáticas, E-mail: ysantiagoa@unmsm.edu.pe

${ }^{3}$ UNMSM, Facultad de Ciencias Matemáticas, E-mail: fparionav@hotmail.com
} 
con $p \geq 2 . \nabla$ es el operador gradiente, div es el operador divergencia, $f_{i}(s, r), i=1,2$, son funciones reales no lineales continuas para $(s, r) \in \mathbb{R}^{2}, w^{\prime}:=\frac{\partial w}{\partial t}, w^{\prime \prime}:=\frac{\partial^{2} w}{\partial t^{2}}$.

Cuando $p=2$, muchos autores estudiaron desde diferentes puntos de vista el sistema $(1,1)-(1,2)$, debemos mencionar: Segal [15], que presentó el significado físico de (1.1) - (1,2): Medeiros y Milla Miranda [9], la existencia y unicidad global; Li y Tsai [6], la existencia, unicidad global, y explosión de soluciones; Wu y Tsai [17], existencia local y explosión de soluciones; Quispe Méndez [13,14], existencia local y explosión de soluciones.

Cuando $p \geq 2$ y $u=v$, las ecuaciones del tipo (1,1) se utilizan para describir el movimiento de un sólido viscoelástico (Por ejemplo, una barra si $n=1$ y una lamina si $n=2$ ) compuesto de un material especial, ver referencias de Yang y Chen [18]. También se puede considerar como una ecuación que gobierna el movimiento longitudinal de una barra viscoelástica obedeciendo el modelo de Voight no lineal [18]. Este tipo de modelos, fueron estudiados por muchos autores, podemos mencionar: Ma y Soriano [8], Gao y Ma [5], Yang y Chen [18], Quispe Méndez [11], Ye [19], Chen, Yao y Shao [3] y entre otros.

Cuando $p \geq 2$, recientemente, Castro [2] probó la existencia de la solución global para el sistema

$$
\begin{aligned}
u^{\prime \prime}-\Delta_{p} u-\Delta u u^{\prime} & =|v|^{\rho+2}|u|^{\rho} u+f_{1}, \\
v^{\prime \prime}-\Delta_{p} v-\Delta v^{\prime} & =|u|^{\rho+2}|v|^{\rho} v+f_{2},
\end{aligned}
$$

donde $\rho \geq-1$. Lima, Lourêdo y Marinho [7] probaron la existencia de una solución local para el sistema

$$
\begin{aligned}
u^{\prime \prime}-\Delta_{p} u-\Delta u^{\prime}+f(u, v) u & =h_{1}, \\
v^{\prime \prime}-\Delta_{p} v-\Delta v^{\prime}+g(u, v) v & =h_{2},
\end{aligned}
$$

donde $f$ es continua en la primera variable y Lipschitziana en la segunda variable y $g$ es Lipschitziana en la primera y continua en la segunda variable. Consecuentemente podemos notar que los sistemas $(1,7)-(1,8)$ y $(1,9)-(1,10)$ son casos particulares del sistema propuesto $(1,1)-(1,2)$

En este trabajo, probaremos la existencia local y la propiedad de explosión de soluciones del problema $(1,1)$ - (1,6) en un dominio acotado $\Omega$ en $\mathbb{R}^{n}$, cuando $f_{i}(s, r), i=1,2$, son funciones reales no lineales continuamente diferenciables para $(s, r) \in \mathbb{R}^{2}$. En primer lugar, probaremos la existencia de una solución local, utilizando el método de Faedo-Galerkin y argumentos de la proyección, compacidad y monotonicidad. En segundo lugar, obtendremos la explosión de soluciones en tiempo finito, con energía inicial negativa, nula y positiva restringida, empleando el método directo desarrollado por Li y Tsai [6]. Asimismo, daremos las estimativas para el tiempo finito de explosión. En la discusión del problema, emplearemos las estrategias y herramientas inspiradas en los trabajos de Li y Tsai [6]. Lima, Lourêdo y Marinho [7], Wu y Tsai [17] y Quispe Méndez [11 - 14] .

\section{Preliminares}

En esta sección presentamos algunas notaciones, conceptos y resultados sin demostración, los cuales serán usados en el desarrollo del presente trabajo.

Sea $\Omega$ un conjunto abierto y acotado de $\mathbb{R}^{n}$ con frontera suficientemente regular $\partial \Omega$. Denotamos el producto interno y la norma de $L^{2}(\Omega)$ y $L^{p}(\Omega)$, con $(.,$.$) y |\cdot|_{p}$, respectivamente, para $1 \leq p \leq \infty$. Además $((.,)$.$) y \|\cdot\|$, denotaran el producto interno y la norma de $H_{0}^{1}(\Omega)$, donde $((u, v)):=$ $\int_{\Omega} \nabla u(x) \cdot \nabla v(x) d x$. En el espacio de Sobolev $W_{0}^{1, p}(\Omega)$ usamos la norma

$$
\|u\|_{1, p}:=\left(\int_{\Omega}|\nabla u(x)|^{p} d x\right)^{\frac{1}{p}} .
$$


Sea $X$ un espacio de Banach, $T$ y $p$ números reales tales que $0<T \leq \infty$ y $1 \leq p \leq \infty$. Representamos con $L^{p}(0 . T ; X)$ al espacio de Banach de las funciones vectoriales $\left.u:\right] 0, T[\longrightarrow X$ medibles con $\|u(t)\|_{X} \in L^{p}(0, T)$, dotado de la norma

$$
\begin{gathered}
\|u\|_{L^{p}(0, T ; X)}:=\left(\int_{0}^{T}\|u(t)\|_{X}^{p} d t\right)^{\frac{1}{p}}, 1 \leq p<\infty, \\
\|u\|_{L^{\infty}(0, T ; X)}:=\sup _{0<t<T} \operatorname{ess}\|u(t)\|_{X}, p=\infty .
\end{gathered}
$$

Similarmente, cuando $0<T<\infty$, representamos con $C([0, T] ; X)$ al espacio de Banach de las funciones continuas $u:[0, T] \longrightarrow X$, dotado de la norma

$$
\|u\|_{C([0, T] ; X)}:=\sup _{0 \leq t \leq T}\|u(t)\|_{X} .
$$

Denotamos $w^{\prime}:=\frac{\partial w}{\partial t}, w^{\prime \prime}:=\frac{\partial^{2} w}{\partial t^{2}}$ y $w(t)(x):=w(x, t)$.

Hipótesis. Imponemos sobre las funciones reales $f_{1}(r, s)$ y $f_{2}(r, s)$ las siguientes condiciones:

(H1) $f_{i} \in C^{1}\left(\mathbb{R}^{2}\right), i=1,2$, y para cada $(u, v) \in H_{0}^{1}(\Omega) \times H_{0}^{1}(\Omega)$, tenemos $u f_{1}(u, v)+v f_{2}(u, v) \in$ $L^{1}(\Omega)$ y $F(u, v) \in L^{1}(\Omega)$, donde

$$
F(u, v):=\int_{0}^{u} f_{1}(\xi, v) d \xi+\int_{0}^{v} f_{2}(0, \xi) d \xi
$$

(H2) Existe una constante positiva $K$ tal que

$$
\left|f_{i}(r, s)\right| \leq K\left(|r|^{\alpha+1}+|s|^{\beta+1}\right),
$$

para cada $\left(r_{1}, s_{1}\right),\left(r_{2}, s_{2}\right) \in \mathbb{R}^{2}, i=1,2$, donde $\frac{p-2}{2} \leq \alpha, \beta \leq \frac{n(p-2)+2 p}{2(n-p)}$ para $1<p<n$ ó $\alpha, \beta \geq$ $\frac{p-2}{2}$ para $1 \leq n \leq p$; para ambos casos $2(\alpha+1)>2(\beta+1) \geq p$ ó $2(\alpha+1) \geq 2(\beta+1)>p$.

(H3) $\frac{\partial f_{1}}{\partial s}(r, s)=\frac{\partial f_{2}}{\partial r}(r, s)$, para cada $(r, s) \in \mathbb{R}^{2}$.

(H4) Existe una constante positiva $\gamma \geq \frac{p-2}{4}$ tal que

$$
r f_{1}(r, s)+s f_{2}(r, s) \geq 2(2 \gamma+1) F(r, s),
$$

para cada $(r, s) \in \mathbb{R}^{2}$, donde $F(r, s)$ es la función dada en $(H 1)$.

Lema 2.1. ([16]). El operador p-Laplaciano se define por

$$
\begin{aligned}
-\Delta_{p}: W_{0}^{1, p}(\Omega) & \rightarrow W^{-1, q}(\Omega) \\
w & \mapsto-\Delta_{p} w
\end{aligned}
$$

donde $\Delta_{p} w:=\operatorname{div}\left(|\nabla w|^{p-2} \nabla w\right), p \geq 2, \frac{1}{p}+\frac{1}{q}=1, y$ tiene las siguientes propiedades:

(i) $-\Delta_{p}$ es monótono, acotado, coercivo y hemicontinuo.

(ii) $\left\langle\left(-\Delta_{p}\right) u, u\right\rangle_{W-1, q(\Omega) \times W_{0}^{1, p}(\Omega)}=\|u\|_{1, p}^{p}$.

(iii) $\left\langle\left(-\Delta_{p}\right) u(t), u^{\prime}(t)\right\rangle_{W^{-1, q}(\Omega) \times W_{0}^{1, p}(\Omega)}=\frac{1}{p} \frac{d}{d t}\|u(t)\|_{1, p}^{p}$.

(iv) $\left\|\left(-\Delta_{p}\right) u\right\|_{W^{-1, q}(\Omega)} \leq C\|u\|_{1, p}^{p-1}$, para alguna constante $C>0$. 
Lema 2.2 (Desigualdad de Sobolev-Poincaré [1]). Si $1 \leq q \leq \frac{n p}{n-p}$ para $1<p<n$ ó $1 \leq q<\infty$ para $1 \leq n \leq p$, entonces existe una constante positiva $B_{1}$ tal que

$$
|u|_{q} \leq B_{1}\|u\|_{1, p}, \forall u \in W_{0}^{1, p}(\Omega) .
$$

Lema 2.3 (Desigualdad Generalizada de Gronwall [10]). Sea $f:[0, \infty[\rightarrow[0, \infty[$ continua, $g:] 0, \infty[\rightarrow] 0, \infty[$ continua y no decreciente y sea $C$ una constante positiva. Si

$$
f(t) \leq C+\int_{0}^{t} g(f(s)) d s, 0 \leq t<\infty
$$

entonces,

$$
f(t) \leq G^{-1}\left(T_{*}\right)<\infty, 0 \leq t \leq T_{*},
$$

para cualquier número fijo $T_{*}<G(\infty)$, donde

$$
G(\tau):=\int_{C}^{\tau} \frac{1}{g(s)} d s, \text { para } \tau \geq C .
$$

Además, si $G(\infty)=\infty$, entonces

$$
f(t) \leq G^{-1}(t), \text { para todo } t \geq 0 .
$$

Lema $2.4([6])$. Sea $\gamma>0$ y sea $B \in C^{2}([0, \infty[)$ una función no negativa que satisface

$$
B^{\prime \prime}(t)-4(\gamma+1) B^{\prime}(t)+4(\gamma+1) B(t) \geq 0
$$

Si $B^{\prime}(0)>r_{2} B(0)+K_{0}$, entonces $B^{\prime}(t)>K_{0}$, para $t>0$, donde $K_{0}$ es una constante $y$

$$
r_{2}:=2(\gamma+1)-2 \sqrt{(\gamma+1) \gamma}
$$

es la menor raíz de la ecuación cuadrática $r^{2}-4(\gamma+1) r+4(\gamma+1)=0$.

Lema $2.5([6])$. Si $J(t)$ es una función no creciente en $\left[t_{0}, \infty\left[, t_{0} \geq 0\right.\right.$ y satisface la inecuación. diferencial

$$
\left[J^{\prime}(t)\right]^{2} \geq a+b[J(t)]^{2+\frac{1}{\gamma}}, \text { para } t \geq t_{0},
$$

donde $a>0, \gamma>0$ y $b \in \mathbb{R}$, entonces existe un número real positivo $T_{*}$ tal que $\lim _{t \rightarrow T_{*}^{-}} J(t)=0$ y una cota superior de $T_{*}$ puede ser estimado respectivamente, en los siguientes casos:

(i) Si $b<0$ y $J\left(t_{0}\right)<\min \left\{1, \sqrt{\frac{a}{-b}}\right\}$, entonces

$$
T_{*} \leq t_{0}+\frac{1}{\sqrt{-b}} \ln \left(\frac{\sqrt{\frac{a}{-b}}}{\sqrt{\frac{a}{-b}}-J\left(t_{0}\right)}\right) .
$$

(ii) Si $b=0$, entonces

$$
T_{*} \leq t_{0}+\frac{J\left(t_{0}\right)}{\sqrt{a}} .
$$


(iii) Si $b>0$, entonces

$$
\begin{gathered}
T_{*} \leq \frac{J\left(t_{0}\right)}{\sqrt{a}} \\
T_{*} \leq t_{0}+2^{\frac{3 \gamma+1}{2 \gamma}} \frac{\gamma c}{\sqrt{a}}\left(1-\left[1+c J\left(t_{0}\right)\right]^{-\frac{1}{2 \gamma}}\right),
\end{gathered}
$$

donde $c:=\left(\frac{b}{a}\right)^{\frac{\gamma}{\gamma+2}}$.

\section{Existencia Local}

En esta sección, discutiremos la existencia de la solución local del problema $(1,1)$ - $(1,6)$, usando el método de Faedo-Galerkin. Así mismo, serán usados los argumentos de la proyección, compacidad y monotonicidad.

Definición 3.1. Un par de funciones $u, v: \Omega \times[0, T[\rightarrow \mathbb{R}$ satisfaciendo

$$
\begin{aligned}
& u, v \in L^{\infty}\left(0, T_{0} ; W_{0}^{1, p}(\Omega)\right), \\
& u^{\prime}, v^{\prime} \in L^{2}\left(0, T_{0} ; H_{0}^{1}(\Omega)\right), \\
& u^{\prime \prime}, v^{\prime \prime} \in L^{2}\left(0, T_{0} ; W^{-1, q}(\Omega)\right),
\end{aligned}
$$

es llamada una solución (débil) del problema $(1,1)-(1,6)$, si verifican las condiciones $(1,3)-(1,6)$ y para todo $w \in W_{0}^{1, p}(\Omega)$, se cumplen las igualdades:

$$
\begin{aligned}
& u^{\prime \prime}-\Delta_{p} u-\Delta u^{\prime}=f_{1}(u, v) \text { en } L^{2}\left(0, T ; W^{-1, q}(\Omega)\right), \\
& v^{\prime \prime}-\Delta_{p} v-\Delta v^{\prime}=f_{2}(u, v) \text { en } L^{2}\left(0, T ; W^{-1, q}(\Omega)\right),
\end{aligned}
$$

donde $p^{-1}+q^{-1}=1$.

Teorema 3.2 (Existencia Local). Supongamos que las funciones $f_{1}$ y $f_{2}$ satisfacen la hipótesis (H2). Si $u_{0}, v_{0} \in W_{0}^{1, p}(\Omega)$ y $u_{1}, v_{1} \in L^{2}(\Omega)$, entonces existe $T_{0}>0$ tal que el problema $(1,1)-(1,6)$ tiene una solución $(u, v)$ en la clase

$$
\begin{aligned}
& u, v \in L^{\infty}\left(0, T_{0} ; W_{0}^{1, p}(\Omega)\right), \\
& u^{\prime}, v^{\prime} \in L^{\infty}\left(0, T_{0} ; L^{2}(\Omega)\right) \cap L^{2}\left(0, T_{0} ; H_{0}^{1}(\Omega)\right), \\
& u^{\prime \prime}, v^{\prime \prime} \in L^{2}\left(0, T_{0} ; W^{-1, q}(\Omega)\right),
\end{aligned}
$$

donde $p^{-1}+q^{-1}=1$.

Demostración. Procedemos en cinco etapas:

Soluciones Aproximadas. Sea $r>n\left(\frac{1}{2}-\frac{1}{p}\right)+1$. Entonces $H_{0}^{r}(\Omega)$ es un espacio de Hilbert separable tal que la inmersión $H_{0}^{r}(\Omega) \hookrightarrow W_{0}^{1, p}(\Omega)$ es continua y densa. En $H_{0}^{r}(\Omega)$, existe una base hilbertiana ortonormal completa $\left\{\omega_{k}\right\}_{k \in \mathbb{N}}$ en $L^{2}(\Omega)$. Consideremos $V_{m}=\left[\omega_{1}, \omega_{2}, \ldots, \omega_{m}\right]$ el subespacio de $H_{0}^{r}(\Omega)$ generado por los primeros $m$ vectores $\omega_{1}, \omega_{2}, \ldots, \omega_{m}$ de $\left\{\omega_{k}\right\}_{k \in \mathbb{N}}$. También tenemos la siguiente cadena de inmersiones continuas y densas

$$
H_{0}^{r}(\Omega) \hookrightarrow W_{0}^{1, p}(\Omega) \hookrightarrow L^{2}(\Omega) \hookrightarrow W^{-1, q}(\Omega) \hookrightarrow H^{-r}(\Omega)
$$

donde $p^{-1}+q^{-1}=1$. Consideremos

$$
u_{m}(t)=\sum_{j=1}^{m} r_{j m}(t) \omega_{j}, v_{m}(t)=\sum_{j=1}^{m} s_{j m}(t) \omega_{j},
$$


las soluciones aproximadas en $V_{m}$ del problema $(1,1)-(1,6)$, donde las funciones $r_{j m}(t), s_{j m}(t)$ $j=1,2 \ldots, m$, son determinadas del siguiente problema en ecuaciones diferenciales ordinarias, para $w \in V_{m}$

donde

$$
\begin{aligned}
& \left(u_{m}^{\prime \prime}(t), w\right)+\left\langle-\Delta_{p} u_{m}(t), w\right\rangle+\left(\left(u_{m}^{\prime}(t), w\right)\right)=\left\langle f_{1}\left(u_{m}, v_{m}\right), w\right\rangle, \\
& \left(v_{m}^{\prime \prime}(t), w\right)+\left\langle-\Delta_{p} v_{m}(t), w\right\rangle+\left(\left(v_{m}^{\prime}(t), w\right)\right)=\left\langle f_{2}\left(u_{m}, v_{m}\right), w\right\rangle, \\
& u_{m}(0)=u_{0 m}, \quad u_{m}^{\prime}(0)=u_{1 m}, \\
& v_{m}(0)=v_{0 m}, \quad v_{m}^{\prime}(0)=v_{1 m},
\end{aligned}
$$

$$
\begin{array}{lll}
u_{0 m}=\sum_{j=1}^{m} r_{0 j m}(t) \omega_{j}, & u_{0 m} \rightarrow u_{0} & \text { fuerte en } W_{0}^{1, p}(\Omega), \\
u_{1 m}=\sum_{j=1}^{m} r_{1 j m}(t) \omega_{j}, & u_{1 m} \rightarrow u_{1} & \text { fuerte en } L^{2}(\Omega), \\
v_{0 m}=\sum_{j=1}^{m} s_{0 j m}(t) \omega_{j}, & v_{0 m} \rightarrow v_{0} & \text { fuerte en } W_{0}^{1, p}(\Omega), \\
v_{1 m}=\sum_{j=1}^{m} s_{1 j m}(t) \omega_{j}, & v_{1 m} \rightarrow v_{1} & \text { fuerte en } L^{2}(\Omega)
\end{array}
$$

El teorema de Carathéodory [4], nos garantiza la existencia de una solución local $\left(u_{m}, v_{m}\right)$ del problema aproximado $(3,2)$ en un intervalo $\left[0, T_{m}[\right.$. Las siguientes estimativas a priori nos permitirán extender la solución $\left(u_{m}, v_{m}\right)$ a un intervalo $[0, T]$, con $T$ independiente de $m$.

Estimativa I. Considerando $w=u_{m}^{\prime}(t)$ en $(3,2)_{1}$ y $w=v_{m}^{\prime}(t)$ en $(3,2)_{2}$, resulta

$$
\begin{aligned}
\frac{1}{2} \frac{d}{d t}\left|u_{m}^{\prime}(t)\right|_{2}^{2}+\frac{1}{p} \frac{d}{d t}\left\|u_{m}(t)\right\|_{1, p}^{p}+\left\|u_{m}^{\prime}(t)\right\|^{2} & =\left\langle f_{1}\left(u_{m}, v_{m}\right), u_{m}^{\prime}\right\rangle, \\
\frac{1}{2} \frac{d}{d t}\left|v_{m}^{\prime}(t)\right|_{2}^{2}+\frac{1}{p} \frac{d}{d t}\left\|v_{m}(t)\right\|_{1, p}^{p}+\left\|v_{m}^{\prime}(t)\right\|^{2} & =\left\langle f_{2}\left(u_{m}, v_{m}\right), v_{m}^{\prime}\right\rangle .
\end{aligned}
$$

Sumando $(3,4)$ y $(3,5)$, obtenemos

donde

$$
\frac{d}{d t} E(t)=\left\langle f_{1}\left(u_{m}, v_{m}\right), u_{m}^{\prime}(t)\right\rangle+\left\langle f_{2}\left(u_{m}, v_{m}\right), v_{m}^{\prime}(t)\right\rangle
$$

$$
\begin{aligned}
E(t):= & \frac{1}{2}\left|u_{m}^{\prime}(t)\right|_{2}^{2}+\frac{1}{2}\left|v_{m}^{\prime}(t)\right|_{2}^{2}+\frac{1}{p}\left\|u_{m}(t)\right\|_{1, p}^{p}+\frac{1}{p}\left\|v_{m}(t)\right\|_{1, p}^{p} \\
& +\int_{0}^{t}\left\|u_{m}^{\prime}(s)\right\|^{2} d s+\int_{0}^{t}\left\|v_{m}^{\prime}(s)\right\|^{2} d s .
\end{aligned}
$$

Ahora, usando la hipótesis (H2), la desigualdad de Hölder, la desigualdad $a b \leq \frac{1}{2}\left(a^{2}+b^{2}\right)$ y la desigualdad de Sobolev-Poincaré, se obtiene

$$
\begin{aligned}
\left\langle f_{1}\left(u_{m}, v_{m}\right), u_{m}^{\prime}(t)\right\rangle & \leq \int_{\Omega}\left|f_{1}\left(u_{m}(x, t), v_{m}(x, t)\right)\right|\left|u_{m}^{\prime}(x, t)\right| d x \\
& \leq K \int_{\Omega}\left[\left|u_{m}(x, t)\right|^{\alpha+1}+\left|v_{m}(x, t)\right|^{\beta+1}\right]\left|u_{m}^{\prime}(x, t)\right| \\
& \leq K\left[\left|u_{m}(t)\right|_{2(\alpha+1)}^{\alpha+1}+\left|v_{m}(t)\right|_{2(\beta+1)}^{\beta+1}\right]\left|u_{m}^{\prime}(t)\right|_{2} \\
& \leq C_{1}\left[\left|u_{m}(t)\right|_{2(\alpha+1)}^{2(\alpha+1)}+\left|v_{m}(t)\right|_{2(\beta+1)}^{2(\beta+1)}+\left|u_{m}^{\prime}(t)\right|_{2}^{2}\right] \\
& \leq C_{2}\left[\left|u_{m}(t)\right|_{1, p}^{2(\alpha+1)}+\left|v_{m}(t)\right|_{1, p}^{2(\beta+1)}+\left|u_{m}^{\prime}(t)\right|_{2}^{2}\right] .
\end{aligned}
$$

Es decir se tiene

$$
\left\langle f_{1}\left(u_{m}, v_{m}\right), u_{m}^{\prime}(t)\right\rangle \leq C_{2}\left[\left|u_{m}(t)\right|_{1, p}^{2(\alpha+1)}+\left|v_{m}(t)\right|_{1, p}^{2(\beta+1)}+\left|u_{m}^{\prime}(t)\right|_{2}^{2}\right],
$$

donde $2(\alpha+1), 2(\beta+1) \leq \frac{n p}{n-p}$. Similarmente, se obtiene 


$$
\left\langle f_{2}\left(u_{m}, v_{m}\right), v_{m}^{\prime}(t)\right\rangle \leq C_{2}\left[\left|u_{m}(t)\right|_{1, p}^{2(\alpha+1)}+\left|v_{m}(t)\right|_{1, p}^{2(\beta+1)}+\left|v_{m}^{\prime}(t)\right|_{2}^{2}\right] .
$$

Sustituyendo $(3,7)-(3,8)$ en $(3,6)$ y utilizando $(3,3)$, obtenemos

$$
\varphi_{m}(t) \leq C_{3}+C_{3} \int_{0}^{t}\left[\varphi_{m}^{\frac{2(\alpha+1)}{p}}(s)+\varphi_{m}^{\frac{2(\beta+1)}{p}}(s)+\varphi_{m}(s)\right] d s,
$$

donde

$$
\begin{aligned}
\varphi_{m}(t): & =\left|u_{m}^{\prime}(t)\right|_{2}^{2}+\left|v_{m}^{\prime}(t)\right|_{2}^{2}+\left\|u_{m}(t)\right\|_{1, p}^{p}+\left\|v_{m}(t)\right\|_{1, p}^{p} \\
& +\int_{0}^{t}\left\|u_{m}^{\prime}(s)\right\|^{2} d s++\int_{0}^{t}\left\|v_{m}^{\prime}(s)\right\|^{2} d s .
\end{aligned}
$$

Consideremos que $\frac{2(\alpha+1)}{p}>\frac{2(\beta+1)}{p} \geq 1$ y definamos la función

$$
G(\tau):=\int_{C_{3}}^{\tau} \frac{d s}{s^{\frac{2(\alpha+1)}{p}}+s^{\frac{2(\beta+1)}{p}}+s}, \text { para } \tau \geq C_{3} .
$$

Entonces

$$
G(\infty) \leq \frac{1}{\left(\frac{2(\alpha+1)}{p}-1\right) C_{3}^{\frac{2(\alpha+1)}{p}-1}} .
$$

Por esta relación y utilizando la desigualdad generalizada de Gronwall, de (3,9), existe un número $T_{0} \in\langle 0, G(\infty)\rangle$ y una constante $K_{0}>0$, independientes de $m$, tal que

$$
\begin{array}{r}
\left\|u_{m}(t)\right\|_{1, p}^{p}+\left\|v_{m}(t)\right\|_{1, p}^{p} \leq K_{0}, \\
\left|u_{m}^{\prime}(t)\right|_{2}^{2}+\left|v_{m}^{\prime}(t)\right|_{2}^{2} \leq K_{0}, \\
\int_{0}^{t}\left\|u_{m}^{\prime}(s)\right\|^{2} d s+\int_{0}^{t}\left\|v_{m}^{\prime}(s)\right\|^{2} d s \leq K_{0},
\end{array}
$$

para todo $t \in\left[0, T_{0}\right]$ y para todo $m \in \mathbb{N}$.

Por el Lema $2.1(i v)$ y $(3,10)$, existe una constante $K_{1}>0$, independiente de $m$, tal que

$$
\left\|\left(-\Delta_{p}\right) u_{m}(t)\right\|_{W^{-1, q}(\Omega)}+\left\|\left(-\Delta_{p}\right) v_{m}(t)\right\|_{W^{-1, q}(\Omega)} \leq K_{1},
$$

para todo $t \in\left[0, T_{0}\right]$ y para todo $m \in \mathbb{N}$, donde $p^{-1}+q^{-1}=1$. También aplicando la hipótesis $(H 2)$, la desigualdad de Sobólev-Poincaré y $(3,10)$, existe una constante $K_{2}>0$, independiente de $m$, tal que

$$
\left|f_{1}\left(u_{m}(t), v_{m}(t)\right)\right|_{2}^{2}+\left|f_{2}\left(u_{m}(t), v_{m}(t)\right)\right|_{2}^{2} \leq K_{2},
$$

para todo $t \in\left[0, T_{0}\right]$ y para todo $m \in \mathbb{N}$.

Estimativa II. Mostraremos que las sucesiones $\left\{u_{m}^{\prime \prime}\right\}_{m \in \mathbb{N}}$ y $\left\{v_{m}^{\prime \prime}\right\}_{m \in \mathbb{N}}$ son acotadas en $L^{\infty}\left(0, T_{0} ; H^{-r}(\Omega)\right)$. Sea $P_{m}: L^{2}(\Omega) \rightarrow V_{m} \subset L^{2}(\Omega)$, el operador proyección en $L^{2}(\Omega)$, definido por

$$
P_{m}(h):=\sum_{j=1}^{m}\left(h, \omega_{j}\right) \omega_{j} .
$$

Observar que tiene las siguientes propiedades: $P_{m} \in \mathcal{L}\left(L^{2}(\Omega)\right), P_{m}=P_{m}^{*}$, donde $P_{m}^{*}$ es el operador adjunto de $P_{m}, P_{m} \in \mathcal{L}\left(H_{0}^{r}(\Omega)\right)$ y $P_{m}(w)=w$, para todo $w \in V_{m}$.

Ahora, de la ecuación aproximada $(3,2)_{1}$ y por la cadena de inmersiones $(3,1)$, tenemos

$$
\left\langle u_{m}^{\prime \prime}(t)-\Delta_{p} u_{m}(t)-\Delta u_{m}^{\prime}(t)-f_{1}\left(u_{m}, v_{m}\right), w\right\rangle_{H^{-r}(\Omega) \times H_{0}^{r}(\Omega)}=0,
$$


para todo $w \in V_{m}$. De esta igualdad, aplicando las propiedades $P_{m}=P_{m}^{*}$ y $P_{m}(w)=w$, para todo $w \in V_{m}$, resulta

$$
P_{m}^{*}\left(u_{m}^{\prime \prime}(t)-\Delta_{p} u_{m}(t)-\Delta u_{m}^{\prime}(t)-f_{1}\left(u_{m}, v_{m}\right)\right)=0
$$

en $V_{m}$. Aplicando el teorema de extensión de Hahn-Banach, se tiene

$$
P_{m}^{*}\left(u_{m}^{\prime \prime}(t)-\Delta_{p} u_{m}(t)-\Delta u_{m}^{\prime}(t)-f_{1}\left(u_{m}, v_{m}\right)\right)=0
$$

en $H_{0}^{r}(\Omega)$. De aquí, por la linealidad de $P_{m}^{*}$ y $u_{m}^{\prime \prime}(t) \in V_{m}$, resulta

$$
u_{m}^{\prime \prime}(t)=-P_{m}^{*}\left(-\Delta_{p} u_{m}(t)\right)-P_{m}^{*}\left(-\Delta u_{m}^{\prime}(t)\right)+P_{m}^{*}\left(f_{1}\left(u_{m}, v_{m}\right)\right)
$$

en $H^{-r}(\Omega)$. Por consiguiente

$$
\begin{aligned}
\left\|u_{m}^{\prime \prime}(t)\right\|_{H^{-r}(\Omega)} \leq & \left\|P_{m}^{*}\left(\left(-\Delta_{p}\right) u_{m}(t)\right)\right\|_{H^{-r}(\Omega)}+\left\|P_{m}^{*}\left((-\Delta) u_{m}^{\prime}(t)\right)\right\|_{H^{-r}(\Omega)} \\
& +\left\|P_{m}^{*}\left(f_{1}\left(u_{m}, v_{m}\right)\right)\right\|_{H^{-r}(\Omega)}
\end{aligned}
$$

Desde que $P_{m} \in \mathcal{L}\left(H_{0}^{r}(\Omega)\right)$, resulta $P_{m}^{*} \in \mathcal{L}\left(H^{-r}(\Omega)\right)$. Por la inmersión $W^{-1, q}(\Omega) \hookrightarrow H^{-r}(\Omega)$ y $(3,13)$, se obtiene $P_{m}^{*} \in \mathcal{L}\left(W^{-1, q}(\Omega), H^{-r}(\Omega)\right)$ y

$$
\begin{aligned}
\left\|P_{m}^{*}\left(\left(-\Delta_{p}\right) u_{m}(t)\right)\right\|_{H^{-r}(\Omega)} & \leq C_{1}\left\|\left(-\Delta_{p}\right) u_{m}(t)\right\|_{W^{-1, q}(\Omega)} \\
& \leq C_{2} .
\end{aligned}
$$

Por $P_{m}^{*} \in \mathcal{L}\left(H^{-r}(\Omega)\right)$, la inmersión $H^{-1}(\Omega) \hookrightarrow H^{-r}(\Omega)$ y $(3,12)$, se obtiene $P_{m}^{*} \in$ $\mathcal{L}\left(H^{-1}(\Omega), H^{-r}(\Omega)\right)$ y

$$
\begin{aligned}
\int_{0}^{t}\left\|P_{m}^{*}\left((-\Delta) u_{m}^{\prime}(s)\right)\right\|_{H^{-r}(\Omega)}^{2} d s & \leq \int_{0}^{t}\left\|(-\Delta) u_{m}^{\prime}(s)\right\|_{H^{-1}(\Omega)}^{2} d s \\
& \leq C_{2} \int_{0}^{t}\left\|u_{m}^{\prime}(s)\right\|^{2} d s \\
& \leq C_{3} .
\end{aligned}
$$

Ahora, acotemos el término $\left\|P_{m}^{*}\left(f_{1}\left(u_{m}(t), v_{m}(t)\right)\right)\right\|_{H^{-r}(\Omega)}$. Desde que $P_{m}^{*} \in \mathcal{L}\left(H^{-r}(\Omega)\right)$, $L^{2}(\Omega) \hookrightarrow W^{-1, q}(\Omega) \hookrightarrow H^{-r}(\Omega)$ y $(3,14)$, se obtiene $P_{m}^{*} \in \mathcal{L}\left(L^{2}(\Omega), H^{-r}(\Omega)\right)$ y

$$
\begin{aligned}
\left\|P_{m}^{*}\left(f_{1}\left(u_{m}, v_{m}\right)\right)\right\|_{H^{-r}(\Omega)} & \leq C_{1}\left|f_{1}\left(u_{m}, v_{m}\right)\right|_{2} \\
& \leq C_{4} .
\end{aligned}
$$

Similarmente, también se obtienen las acotaciones para $\left\{v_{m}^{\prime \prime}\right\}_{m \in \mathbb{N}}$. Por tanto, sustituyendo $(3,16),(3,17)$ y $(3,18)$ en $(3,15)$, se obtiene una constante $K_{3}>0$, independiente de $m$, tal que

$$
\int_{0}^{t}\left\|u_{m}^{\prime \prime}(s)\right\|_{H^{-r}(\Omega)}^{2} d s+\int_{0}^{t}\left\|v_{m}^{\prime \prime}(s)\right\|_{H^{-r}(\Omega)}^{2} d s \leq K_{3},
$$

para todo $t \in\left[0, T_{0}\right]$ y para todo $m \in \mathbb{N}$.

Pasaje al límite. De las estimativas $(3,10)-(3,12)$, existen subsucesiones $\left\{u_{\nu}\right\},\left\{u_{\nu}^{\prime}\right\}$ y $\left\{u_{\nu}^{\prime \prime}\right\}$ de $\left\{u_{m}\right\}$, $\left\{u_{m}^{\prime}\right\}$ y $\left\{u_{m}^{\prime \prime}\right\}$, respectivamente, tales que 


$$
\begin{array}{cll}
u_{\nu} \stackrel{*}{\rightarrow} u & \text { en } & L^{\infty}\left(\left[0, T_{0}\right] ; W_{0}^{1, p}(\Omega)\right) \\
u_{\nu}^{\prime} * u^{\prime} & \text { en } & L^{\infty}\left(\left[0, T_{0}\right] ; L^{2}(\Omega)\right), \\
u_{\nu}^{\prime} \rightarrow u^{\prime} & \text { en } & L^{2}\left(0, T_{0} ; H_{0}^{1}(\Omega)\right), \\
u_{\nu}^{\prime \prime} \rightarrow u^{\prime \prime} & \text { en } & L^{2}\left(0, T_{0} ; H^{-r}(\Omega)\right), \\
\left(-\Delta_{p}\right) u_{\nu} \stackrel{*}{\rightarrow} \chi_{1} & \text { en } & L^{\infty}\left(0, T_{0} ; W^{-1, q}(\Omega)\right), \\
& & \\
v_{\nu} \stackrel{*}{*} v & \text { en } & L^{\infty}\left(\left[0, T_{0}\right] ; W_{0}^{1, p}(\Omega)\right) \\
v_{\nu}^{\prime} * v^{\prime} & \text { en } & L^{\infty}\left(\left[0, T_{0}\right] ; L^{2}(\Omega)\right), \\
v_{\nu}^{\prime} \rightarrow v^{\prime} & \text { en } & L^{2}\left(0, T_{0} ; H_{0}^{1}(\Omega)\right), \\
v_{\nu}^{\prime \prime} \rightarrow v^{\prime \prime} & \text { en } & L^{2}\left(0, T_{0} ; H^{-r}(\Omega)\right), \\
\left(-\Delta_{p}\right) v_{\nu} \stackrel{*}{*} \chi_{2} & \text { en } & L^{\infty}\left(0, T_{0} ; W^{-1, q}(\Omega)\right),
\end{array}
$$

Desde que se cumplen las inmersiones $W_{0}^{1, p}(\Omega) \stackrel{c}{\hookrightarrow} L^{2}(\Omega), \quad L^{\infty}\left(0, T_{0} ; W_{0}^{1, p}(\Omega)\right) \hookrightarrow$ $L^{2}\left(0, T_{0} ; W_{0}^{1, p}(\Omega)\right)$ y $L^{\infty}\left(0, T_{0} ; L^{2}(\Omega)\right) \hookrightarrow L^{2}\left(0, T_{0} ; L^{2}(\Omega)\right)$, aplicando el lema de compacidad de Lions-Aubin, de $(3,10)$ y $(3,12)$, resultan

$$
\begin{array}{ccc}
u_{\nu} \rightarrow u & \text { en } & L^{2}\left(\left[0, T_{0}\right] ; L^{2}(\Omega)\right), \\
v_{\nu} \rightarrow v & \text { en } & L^{2}\left(\left[0, T_{0}\right] ; L^{2}(\Omega)\right),
\end{array}
$$

y

$$
\begin{array}{lll}
u_{\nu} \rightarrow u & c . t . p . \text { en } & \Omega \times\left[0, T_{0}\right], \\
v_{\nu} \rightarrow v & \text { c.t.p. en } & \Omega \times\left[0, T_{0}\right] .
\end{array}
$$

Usando $(3.14)$ y $(3,23)$ vemos que

$$
\int_{0}^{T_{0}}\left|f_{1}\left(u_{\nu}(t), v_{\nu}(t)\right)\right|_{2}^{2} d t+\int_{0}^{T_{0}}\left|f_{2}\left(u_{\nu}(t), v_{\nu}(t)\right)\right|_{2}^{2} d t \leq C
$$

y

$$
\begin{aligned}
& f_{1}\left(u_{\nu}, v_{\nu}\right) \rightarrow f_{1}(u, v) \quad \text { c.t.p. en } \Omega \times\left[0, T_{0}\right], \\
& f_{2}\left(u_{\nu}, v_{\nu}\right) \rightarrow f_{2}(u, v) \quad \text { c.t.p. en } \Omega \times\left[0, T_{0}\right] .
\end{aligned}
$$

Por $(3,24)-(3,25)$ y el lema de Lions, se deduce que

$$
\begin{aligned}
& f_{1}\left(u_{\nu}, v_{\nu}\right) \rightarrow f_{1}(u, v) \text { en } L^{2}\left(\left[0, T_{0}\right] ; L^{2}(\Omega)\right) \text {, } \\
& f_{2}\left(u_{\nu}, v_{\nu}\right) \rightarrow f_{2}(u, v) \text { en } L^{2}\left(\left[0, T_{0}\right] ; L^{2}(\Omega)\right) \text {. }
\end{aligned}
$$

De las convergencias $(3,20)$ - $(3,21)$ y $(3,26)$ por pasaje al límite en la ecuación aproximada $(3,2)$, resulta

$$
\begin{aligned}
\frac{d}{d t}\left(u^{\prime}(t), w\right)+\left\langle\chi_{1}(t), w\right\rangle+\left(\left(u^{\prime}(t), w\right)\right) & =\left(f_{1}(u(t), v(t)), w\right), \\
\frac{d}{d t}\left(v^{\prime}(t), w\right)+\left\langle\chi_{1}(t), w\right\rangle+\left(\left(v^{\prime}(t), w\right)\right) & =\left(f_{2}(u(t), v(t)), w\right),
\end{aligned}
$$

para todo $w \in W_{0}^{1, p}(\Omega)$, en el sentido de las distribuciones $\mathcal{D}^{\prime}\left(0, T_{0}\right)$. De aquí se obtiene

$$
\begin{array}{ccc}
u^{\prime \prime}+\chi_{1}-\Delta u^{\prime}=f_{1}(u, v) & \text { en } & \mathcal{D}^{\prime}\left(\Omega \times\left(0, T_{0}\right)\right), \\
v^{\prime \prime}+\chi_{2}-\Delta v^{\prime}=f_{2}(u, v) & \text { en } & \mathcal{D}^{\prime}\left(\Omega \times\left(0, T_{0}\right)\right) .
\end{array}
$$

Como se cumplen $(-\Delta) u^{\prime},(-\Delta) v^{\prime} \in L^{2}\left(0, T_{0} ; H^{-1}(\Omega)\right) ; f_{1}, f_{2} \in L^{2}\left(\left[0, T_{0}\right], L^{2}(\Omega)\right) ; \chi_{1}, \chi_{2} \in$ $L^{\infty}\left(0, T_{0} ; W^{-1, q}(\Omega)\right) \hookrightarrow L^{2}\left(0, T_{0} ; W^{-1, q}(\Omega)\right)$ y de las inmersiones continuas

$$
W_{0}^{1, p}(\Omega) \hookrightarrow H_{0}^{1}(\Omega) \hookrightarrow L^{2}(\Omega) \hookrightarrow H^{-1}(\Omega) \hookrightarrow W^{-1, q}(\Omega),
$$


resulta $u^{\prime \prime}, v^{\prime \prime} \in L^{2}\left(0, T_{0} ; W^{-1, q}(\Omega)\right)$. Por tanto, de $(3,27)$ se obtiene

$$
\begin{aligned}
& u^{\prime \prime}+\chi_{1}-\Delta u^{\prime}=f_{1}(u, v) \text { en } L^{2}\left(0, T_{0} ; W^{-1, q}(\Omega)\right), \\
& v^{\prime \prime}+\chi_{2}-\Delta v^{\prime}=f_{2}(u, v) \text { en } L^{2}\left(0, T_{0} ; W^{-1, q}(\Omega)\right) .
\end{aligned}
$$

Probemos que $\chi_{1}=\left(-\Delta_{p}\right) u$ y $\chi_{2}=\left(-\Delta_{p}\right) v$. Sea $\mathcal{A}:=-\Delta_{p}$. Tomando $m=\nu$ y $w=u_{\nu}$ en $(3,2)_{1}$ e integrando sobre $[0, t]$, obtenemos

$$
\begin{aligned}
\int_{0}^{t}\left\langle\mathcal{A} u_{\nu}(s), u_{\nu}(s)\right\rangle d s= & \int_{0}^{t}\left(f_{1}\left(u_{\nu}(s), v_{\nu}(s)\right), u_{\nu}(s)\right) d s \\
& -\int_{0}^{t}\left(u_{\nu}^{\prime \prime}(s), u_{\nu}(s)\right) d s \\
& -\frac{1}{2}\left\|u_{\nu}(t)\right\|^{2}+\frac{1}{2}\left\|u_{0 \nu}\right\|^{2} .
\end{aligned}
$$

Por $(3,3)_{1},(3,3)_{2},(3,20)_{1},(3,20)_{2}$ y $(3,20)_{3}$, resulta

$$
\begin{aligned}
\int_{0}^{t}\left(u_{\nu}^{\prime \prime}(s), u_{\nu}(s)\right) d s & =\left(u_{\nu}^{\prime}(t), u_{\nu}(t)\right)-\left(u_{1 \nu}, u_{0 \nu}\right)-\int_{0}^{t}\left|u_{\nu}^{\prime}(s)\right|_{2}^{2} d s \\
& \rightarrow\left(u^{\prime}(t), u(t)\right)-\left(u_{1}, u_{0}\right)-\int_{0}^{t}\left|u^{\prime}(s)\right|_{2}^{2} d s \\
& =\int_{0}^{t}\left(u^{\prime \prime}(s), u(s)\right) d s .
\end{aligned}
$$

También por $W_{0}^{1, p}(\Omega) \hookrightarrow L^{2}(\Omega)$. $(3,20)_{1},(3,21)_{1}$ y $(3,26)_{1}$, se obtiene

$$
\begin{array}{lll}
u_{\nu}(t) \hookrightarrow u(t) & \text { en } & L^{2}(\Omega), \\
v_{\nu}(t) \hookrightarrow v(t) & \text { en } & L^{2}(\Omega)
\end{array}
$$

y

$$
\left(f_{1}\left(u_{\nu}(t), v_{\nu}(t)\right), u_{\nu}(t)\right) \rightarrow\left(f_{1}(u(t), v(t)), u(t)\right),
$$

para c.t.p. $t \in\left[0, T_{0}\right]$. Por hipótesis $(H 2), W_{0}^{1, p}(\Omega) \hookrightarrow L^{2}(\Omega),(3,10)$ y $(3,14)$, resulta

$$
\begin{aligned}
\left|\left(f_{1}\left(u_{\nu}(t), v_{\nu}(t)\right), u_{\nu}(t)\right)\right| & \leq\left|f_{1}\left(u_{\nu}(t), v_{\nu}(t)\right)\right|_{2}\left|u_{\nu}(t)\right|_{2} \\
& \leq C
\end{aligned}
$$

para $t \in\left[0, T_{0}\right]$. De $(3,31),(3,32)$ y el teorema de convergencia dominada de Lebesgue, resulta

$$
\int_{0}^{t}\left(f_{1}\left(u_{\nu}(s), v_{\nu}(s)\right), u_{\nu}(s)\right) d s \rightarrow \int_{0}^{t}\left(f_{1}(u(s), v(s)), u(s)\right) d s
$$

para $t \in\left[0, T_{0}\right]$. Por pasaje al límite en (3.29), haciendo uso de $(3.3)_{1},(3.20)_{1},(3.30)$, (3.33) y $(3.28)_{1}$, obtenemos 


$$
\begin{aligned}
\lim _{\nu \rightarrow \infty} \sup \int_{0}^{t}\left\langle\mathcal{A} u_{\nu}(s), u_{\nu}(s)\right\rangle d s \leq & \int_{0}^{t} \lim _{\nu \rightarrow \infty} \sup \left\langle\mathcal{A} u_{\nu}(s), u_{\nu}(s)\right\rangle d s \\
= & \int_{0}^{t}\left(f_{1}(u(s), v(s)), u(s)\right) d s \\
& -\int_{0}^{t}\left(u^{\prime \prime}(s), u(s)\right) d s \\
& -\frac{1}{2}\|u(t)\|^{2}+\frac{1}{2}\left\|u_{0}\right\|^{2} \\
= & \int_{0}^{t}\left\langle\chi_{1}(s), u(s)\right\rangle d s .
\end{aligned}
$$

Para cada $w \in L^{2}\left(0, T_{0} ; W_{0}^{1, p}(\Omega)\right)$, definamos la función

$$
\psi_{\nu}(t):=\int_{0}^{t}\left\langle\mathcal{A} u_{\nu}(s)-\mathcal{A} w(s), u_{\nu}(s)-w(s)\right\rangle d s, \text { para } t \in\left[0, T_{0}\right] .
$$

Por la monotonía del operador $\mathcal{A}, \psi(t) \geq 0$, para $t \in\left[0, T_{0}\right]$. Por $(3,34),(3,20)_{1}$ y $(3,20)_{5}$, resulta

$$
\begin{aligned}
0 & \leq \lim _{\nu \rightarrow \infty} \sup \psi_{\nu}(t) \\
& \leq \int_{0}^{t} \lim _{\nu \rightarrow \infty} \sup \left\langle\mathcal{A} u_{\nu}(s)-\mathcal{A} w(s), u_{\nu}(s)-w(s)\right\rangle d s \\
& =\int_{0}^{t}\left\langle\chi_{1}(s)-\mathcal{A} w(s), u(s)-w(s)\right\rangle d s .
\end{aligned}
$$

Escogiendo $w=u-\lambda z$ en $(3,35)$, donde $\lambda>0, z \in L^{2}\left(0, T_{0} ; W_{0}^{1, p}(\Omega)\right)$, se tiene

$$
\int_{0}^{t}\left\langle\chi_{1}(s)-\mathcal{A}(u(s)-\lambda z(s)), z(s)\right\rangle d s \geq 0 .
$$

Desde que $\mathcal{A}$ es un operador hemicontinuo y haciendo tender $\lambda \rightarrow 0$, conseguimos

$$
\int_{0}^{t}\left\langle\chi_{1}(s)-\mathcal{A} u(s), z(s)\right\rangle d s \geq 0, \text { para todo } z \in L^{2}\left(0, T_{0} ; W_{0}^{1, p}(\Omega)\right) .
$$

De aquí resulta $\chi_{1}=\mathcal{A} u$. Del mismo modo se obtiene $\chi_{2}=\mathcal{A} v$.

Los datos iniciales se verifican de modo estandar.

Esto concluye la demostración del Teorema 3.2.

\section{Explosión de Soluciones}

En esta sección, discutiremos la propiedad de explosión de soluciones en tiempo finito del problema (1.1)-(1.6). En la discusión usaremos el método directo, utilizado por Li y Tsai [6].

Definición 4.1. Una solución $(u, v)$ del problema $(1,1)-(1,6)$ es llamada explosión si existe un número finito $T_{*}$ tal que

$$
\lim _{t \rightarrow T_{*}^{-}} \int_{\Omega}\left(|\nabla u(x, t)|^{2}+|\nabla v(x, t)|^{2}\right) d x=\infty .
$$

Definición 4.2. La función energía $E(t)$ del problema (1.1)-( 1.6) se define por

$$
\begin{aligned}
E(t):= & \frac{1}{2}\left[\left|u^{\prime}(t)\right|_{2}^{2}+\left|v^{\prime}(t)\right|_{2}^{2}\right]+\frac{1}{p}\left[\|u(t)\|_{1, p}^{p}+\|v(t)\|_{1, p}^{p}\right] \\
& -\int_{\Omega} F(u(x, t), v(x, t)) d x,
\end{aligned}
$$


para $t \geq 0$. donde

$$
F(r, s):=\int_{0}^{r} f_{1}(\xi, s) d \xi+\int_{0}^{s} f_{2}(0, \xi) d \xi
$$

Lema 4.3. Supongamos que se cumplen las hipótesis $(H 1)-(H 3)$. Si $(u, v)$ es una solución del problema (1.1)-(1.6) con datos iniciales $u_{0}, v_{0} \in W_{0}^{1, p}(\Omega)$ y $u_{1}, v_{1} \in L^{2}(\Omega)$, entonces

$$
E(t)+\int_{0}^{t}\left[\left\|u^{\prime}(s)\right\|^{2}+\left\|v^{\prime}(s)\right\|^{2}\right] d s=E(0),
$$

para $t \geq 0$, donde $E(0)$ es la energía inicial definida por

$$
\begin{aligned}
E(0):= & \frac{1}{2}\left[\left|u_{1}\right|_{2}^{2}+\left|v_{1}\right|_{2}^{2}\right]+\frac{1}{p}\left[\left\|u_{0}\right\|_{1, p}^{p}+\left\|v_{0}\right\|_{1, p}^{p}\right] \\
& -\int_{\Omega} F\left(u_{0}(x), v_{0}(x)\right) d x .
\end{aligned}
$$

Demostración. Multiplicando a la ecuación (1.1) por $u^{\prime}$ y a la ecuación $(1,2)$ por $v^{\prime}$, sumando estos resultados, integrando sobre $\Omega$, utilizando el teorema de la Divergencia, el Lema 2.1, (H1) y (H3), obtenemos

$$
E^{\prime}(t)+\left[\left\|u^{\prime}(t)\right\|^{2}+\left\|v^{\prime}(t)\right\|^{2}\right]=0
$$

De aquí, se obtiene el resultado.

Definición 4.4. Para una solución $(u, v)$ del problema (1.1)-( 1.6) definimos la función explosión

$$
A(t):=\left[|u(t)|_{2}^{2}+|v(t)|_{2}^{2}\right]+\int_{0}^{t}\left[\|u(s)\|^{2}+\|v(s)\|^{2}\right] d s, \text { para } t \geq 0 .
$$

Lema 4.5. Supongamos que se cumplen las hipótesis $(H 1)-(H 4)$. Si $(u, v)$ es una solución del problema $(1,1)-(1,6)$ con datos iniciales $u_{0}, v_{0} \in H_{0}^{1}(\Omega) \cap H^{2}(\Omega)$ y $u_{1}, v_{1} \in L^{2}(\Omega)$, entonces

$$
\begin{aligned}
A^{\prime \prime}(t) & -4(\gamma+1)\left[\left|u^{\prime}(t)\right|_{2}^{2}+\left|v^{\prime}(t)\right|_{2}^{2}\right. \\
& \left.+\int_{0}^{t}\left[\left\|u^{\prime}(s)\right\|^{2}+\left\|v^{\prime}(s)\right\|^{2}\right] d s\right] \geq-4(2 \gamma+1) E(0),
\end{aligned}
$$

para. $t \geq 0$, donde $\gamma$ es la constante dada en $(H 4)$.

Demostración. Por diferenciación de $(4,2)$, se tiene

$$
A^{\prime}(t)=2\left[\left(u^{\prime}(t), u(t)\right)+\left(v^{\prime}(t), v(t)\right)\right]+\left[\|u(t)\|^{2}+\|v(t)\|^{2}\right] .
$$

Luego diferenciando (4.4), utilizando las ecuaciones (1.1) -( 1.2) y el teorema de la Divergencia, se obtiene

$$
\begin{aligned}
A^{\prime \prime}(t)= & 2\left[\left|u^{\prime}(t)\right|_{2}^{2}+\left|v^{\prime}(t)\right|_{2}^{2}\right]-2\left[\|u(t)\|_{1, p}^{p}+\|v(t)\|_{1, p}^{p}\right] \\
& +2\left[\left(f_{1}(u(t), v(t)), u(t)\right)+\left(f_{2}(u(t), v(t)), v(t)\right)\right]
\end{aligned}
$$

De (4.5) y (4.1), resulta 


$$
\begin{aligned}
& A^{\prime \prime}(t)-4(\gamma+1)\left[\left|u^{\prime}(t)\right|_{2}^{2}+\left|v^{\prime}(t)\right|_{2}^{2}\right. \\
& \left.+\int_{0}^{t}\left[\left\|u^{\prime}(s)\right\|^{2}+\left\|v^{\prime}(s)\right\|^{2}\right] d s\right]=-4(2 \gamma+1) E(0) \\
& \quad+\frac{2}{p}(4 \gamma+2-p)\left[\|u(t)\|_{1, p}^{p}+\|v(t)\|_{1, p}^{p}\right] \\
& \quad+2 \int_{\Omega}\left[u f_{1}(u, v)+v f_{2}(u, v)-2(2 \gamma+1) F(u, v)\right] d x \\
& \quad+4 \gamma\left[\int_{0}^{t}\left\|u^{\prime}(s)\right\|^{2} d s+\int_{0}^{t}\left\|v^{\prime}(s)\right\|^{2} d s\right] .
\end{aligned}
$$

Empleando las hipótesis $(H 1)$ y $(H 4)$, y utilizando (4.6), se obtiene la relación (4.3) .

Lema 4.6. Supongamos que se cumplen las hipótesis $(H 1)-(H 4)$. Si $(u, v)$ es una solución del problema $(1,1)-(1,6)$ con datos iniciales $u_{0}, v_{0} \in W_{0}^{1, p}(\Omega)$ y $u_{1}, v_{1} \in L^{2}(\Omega)$, y satisfaciendo una de las siguientes condiciones:

(i) $E(0)<0$,

(ii) $E(0)=0$ y $A^{\prime}(0)>K_{0}$,

(iii) $E(0)>0 \quad$ y $A^{\prime}(0)>r_{2}\left[A(0)+\frac{K_{1}}{4(\gamma+1)}\right]+K_{0}$,

donde

$$
\begin{gathered}
K_{0}:=\left\|u_{0}\right\|^{2}+\left\|v_{0}\right\|^{2}, \\
A(0):=\left|u_{0}\right|_{2}^{2}+\left|v_{0}\right|_{2}^{2}, \quad A^{\prime}(0):=2\left[\left(u_{1}, u_{0}\right)+\left(v_{1}, v_{0}\right)\right]+K_{0}, \\
K_{1}:=4(2 \gamma+1) E(0)+4(\gamma+1) K_{0}, \\
r_{2}:=2(\gamma+1)-2 \sqrt{(\gamma+1) \gamma},
\end{gathered}
$$

entonces

$$
A^{\prime}(t)>K_{0}, \text { para } t>t_{0},
$$

donde $t_{0}:=\operatorname{máx}\left\{\frac{A^{\prime}(0)-K_{0}}{4(1+2 \gamma) E(0)}, 0\right\}$ en el caso $(i)$ y $t_{0}:=0$ en los casos (ii) y (iii).

Demostración. Consideremos tres casos de acuerdo al signo de la energía inicial $E(0)$.

(i) $\mathrm{Si} E(0)<0$, de (4.3), se tiene

$$
A^{\prime \prime}(t) \geq-4(2 \gamma+1) E(0)
$$

e integrando, resulta

$$
A^{\prime}(t) \geq A^{\prime}(0)-4(2 \gamma+1) E(0) t, \text { para } t \geq 0 .
$$

Considerando $A^{\prime}(0)-K_{0}-4(2 \gamma+1) E(0) t>0$, se obtiene

$$
A^{\prime}(t)>K_{0} \text {, para } t>t_{0}
$$

donde

$$
t_{0}:=\operatorname{máx}\left\{\frac{A^{\prime}(0)-K_{0}}{4(1+2 \gamma) E(0)}, 0\right\} \text {. }
$$

(ii) Si $E(0)=0$, de $(4,3)$, se tiene

$$
A^{\prime \prime}(t) \geq 0
$$

e integrando, resulta

$$
A^{\prime}(t) \geq A^{\prime}(0), \text { para } t \geq 0 .
$$

Considerando $A^{\prime}(0)-K_{0}>0$, se obtiene

$$
A^{\prime}(t)>K_{0}, \text { para } t \geq 0 .
$$

(iii) Para $E(0)>0$. Primero notemos que se cumple 


$$
2 \int_{0}^{t}\left(\left(w^{\prime}(s), w(s)\right)\right) d s=\|w(t)\|^{2}-\left\|w_{0}\right\|^{2} .
$$

Usando la desigualdad de Hölder en (4.9), se obtiene

$$
\|w(t)\|^{2} \leq\left\|w_{0}\right\|^{2}+\int_{0}^{t}\|w(s)\|^{2} d s+\int_{0}^{t}\left\|w^{\prime}(s)\right\|^{2} d s .
$$

Nuevamente usando la desigualdad de Hölder en (4.4) y por (4.10), resulta

$$
\begin{aligned}
A^{\prime}(t) \leq & A(t)+K_{0}+\left[\left|u^{\prime}(t)\right|_{2}^{2}+\left|v^{\prime}(t)\right|_{2}^{2}\right] \\
& +\int_{0}^{t}\left[\left\|u^{\prime}(s)\right\|_{2}^{2}+\left\|v^{\prime}(s)\right\|_{2}^{2}\right] d s .
\end{aligned}
$$

De (4.3) y (4.11), obtenemos

$$
A^{\prime \prime}(t)-4(\gamma+1) A^{\prime}(t)+4(\gamma+1) A(t)+K_{1} \geq 0
$$

donde

$$
K_{1}:=4(2 \gamma+1) E(0)+4(\gamma+1) K_{0}
$$

Definamos la función

$$
B(t):=A(t)+\frac{K_{1}}{4(\gamma+1)}, \text { para } t \geq 0
$$

Considerando $B^{\prime}(0)>r_{2} B(0)+K_{0}$, la función $B$ satisface las condiciones del Lema 2.4. Así se tiene $A^{\prime}(t)>K_{0}$, para $t>0$. Con esto se concluye la prueba del Lema 4.6.

Definición 4.7. Para las estimativas del tiempo finito de la función explosión $A(t)$, definamos la función

$$
J(t):=\left[A(t)+\left(T_{1}-t\right) K_{0}\right]^{-\gamma}, \text { para } t \in\left[0, T_{1}\right],
$$

donde $T_{1}$ es una constante positiva que se determina posteriormente y $\gamma$ es la constante dada en $(H 4)$.

Teorema 4.8 (Explosión de Soluciones). Supongamos que se cumplen las hipótesis $(H 1)-(H 4)$. Si $(u, v)$ es una solución del problema $(1,1)-(1,6)$ con datos iniciales $u_{0}, v_{0} \in W_{0}^{1, p}(\Omega)$ y $u_{1}, v_{1} \in L^{2}(\Omega)$, y satisfaciendo una de las siguientes condiciones:

(i) $E(0)<0$,

(ii) $E(0)=0 \quad$ y $\quad A^{\prime}(0)>K_{0}$,

(iii) $0<E(0)<\frac{\left[A^{\prime}(0)-K_{0}\right]^{2}}{8\left[A(0)+T_{1} K_{0}\right]}$ y $\quad A^{\prime}(0)>r_{2}\left[A(0)+\frac{K_{1}}{4(\gamma+1)}\right]+K_{0}$,

entonces $(u, v)$ es una solución explosión en tiempo finito $T_{*}$. Además el tiempo finito $T_{*}$ es estimado, en el caso $(i)$,

$$
T_{*} \leq t_{0}-\frac{J\left(t_{0}\right)}{J^{\prime}\left(t_{0}\right)}
$$

Además, si $J\left(t_{0}\right)<\operatorname{mín}\left\{1, \sqrt{\frac{a}{-b}}\right\}$, entonces

$$
T_{*} \leq t_{0}+\frac{1}{\sqrt{-b}} \ln \left(\frac{\sqrt{\frac{a}{-b}}}{\sqrt{\frac{a}{-b}}-J\left(t_{0}\right)}\right) .
$$


En el caso $(i i)$,

$$
T_{*} \leq t_{0}-\frac{J\left(t_{0}\right)}{J^{\prime}\left(t_{0}\right)}
$$

o

$$
T_{*} \leq t_{0}+\frac{J\left(t_{0}\right)}{\sqrt{a}}
$$

En el caso (iii),

$$
T_{*} \leq \frac{J\left(t_{0}\right)}{\sqrt{a}}
$$

o

$$
T_{*} \leq t_{0}+2^{\frac{3 \gamma+1}{2 \gamma}} \frac{\gamma c}{\sqrt{a}}\left\{1-\left[1+c J\left(t_{0}\right)\right]^{-\frac{1}{2 \gamma}}\right\},
$$

donde $a:=\gamma^{2}\left[J\left(t_{0}\right)\right]^{\frac{2}{\gamma}+2}\left[\left[A^{\prime}\left(t_{0}\right)-K_{0}\right]^{2}-8 E(0)\left[J\left(t_{0}\right)\right]^{\frac{-1}{\gamma}}\right], b:=8 \gamma^{2} E(0)$ y $c:=\left(\frac{b}{a}\right)^{\frac{\gamma}{\gamma+2}}$. En el caso $(i), t_{0}:=\operatorname{máx}\left\{\frac{A^{\prime}(0)-K_{0}}{4(1+2 \gamma) E(0)}, 0\right\}$ y $t_{0}:=0$ en los casos (ii) y (iii).

Demostración. Por diferenciación de $(4,12)$, resulta

$$
J^{\prime}(t)=-\gamma[J(t)]^{\frac{1}{\gamma}+1}\left[A^{\prime}(t)-K_{0}\right]
$$

y

$$
J^{\prime \prime}(t)=-\gamma[J(t)]^{\frac{2}{\gamma}+1} V(t)
$$

donde

$$
V(t):=A^{\prime \prime}(t)\left[A(t)+\left(T_{1}-t\right) K_{0}\right]-(\gamma+1)\left[A^{\prime}(t)-K_{0}\right]^{2} .
$$

Utilizando la desigualdad $\left(\sum_{i=1}^{4} a_{i} b_{i}\right)^{2} \leq\left(\sum_{i=1}^{4} a_{i}^{2}\right)\left(\sum_{i=1}^{4} b_{i}^{2}\right),(4,9)$ y la desigualdad de Hölder, de $(4,4)$, resulta

$$
\begin{aligned}
{\left[A^{\prime}(t)-K_{0}\right]^{2} \leq } & 4\left[A(t)+\left(T_{1}-t\right) K_{0}\right]\left[\left|u^{\prime}(t)\right|_{2}^{2}+\left|v^{\prime}(t)\right|_{2}^{2}\right. \\
& \left.+\int_{0}^{t}\left[\left\|u^{\prime}(s)\right\|_{2}^{2}+\left\|v^{\prime}(s)\right\|_{2}^{2}\right] d s\right]
\end{aligned}
$$

De $(4,20)$ y $(4,21)$. se tiene

$$
J^{\prime \prime}(t) \leq-\gamma[J(t)]^{\frac{1}{\gamma}+1} K(t)
$$

donde

$$
\begin{aligned}
K(t):=A^{\prime \prime}(t)-4(\gamma+1)\left[\left|u^{\prime}(t)\right|_{2}^{2}+\left|v^{\prime}(t)\right|_{2}^{2}\right. & \\
& \left.+\int_{0}^{t}\left[\left\|u^{\prime}(s)\right\|^{2}+\left\|v^{\prime}(s)\right\|_{2}^{2}\right] d s\right] .
\end{aligned}
$$

Por $(4,3)$ y $(4,22)$, resulta

$$
J^{\prime \prime}(t) \leq 4 \gamma(2 \gamma+1) E(0)[J(t)]^{\frac{1}{\gamma}+1}, \text { para } t \geq t_{0}
$$


De $(4,8)$ y $(4,19)$, se tiene

$$
J^{\prime}(t)<0, \text { para } t>t_{0} .
$$

Multiplicando $(4,23)$ por $J^{\prime}(t)$ y luego integrando de $t_{0}$ a $t$, se obtiene

$$
\left[J^{\prime}(t)\right]^{2} \geq a+b[J(t)]^{2+\frac{1}{\gamma}}, \text { para } t \geq t_{0}
$$

donde

$$
\begin{aligned}
a & :=\left[J^{\prime}\left(t_{0}\right)\right]^{2}-8 \gamma^{2} E(0)\left[J\left(t_{0}\right)\right]^{\frac{1}{\gamma}+2} \\
& =\gamma^{2}\left[J\left(t_{0}\right)\right]^{\frac{2}{\gamma}+2}\left[\left[A^{\prime}\left(t_{0}\right)-K_{0}\right]^{2}-8 E(0)\left[J\left(t_{0}\right)\right]^{\frac{-1}{\gamma}}\right]
\end{aligned}
$$

y

$$
b:=8 \gamma^{2} E(0)
$$

Observemos que $a>0$ si, y sólo si, $E(0)<\frac{\left[A^{\prime}\left(t_{0}\right)-K_{0}\right]^{2}}{8\left[A\left(t_{0}\right)+\left(T_{1}-t_{0}\right) K_{0}\right]}$.

El caso particular en el que $E(0)<0$, por $(4,23)$ y $(4,24)$, se obtiene directamente $\lim _{t \rightarrow T_{*}^{-}} J(t)=0$ y la estimativa $(4,13)$ para el tiempo finito $T_{*}$. Para los demás casos, por $(4,24)$ y $(4,25)$, la función $J$ satisface las condiciones del Lema 2.5. Entonces existe un tiempo finito $T_{*}$ tal que $\lim _{t \rightarrow T_{*}^{-}} J(t)=0$ y la cota superior para $T_{*}$ son estimadas respectivamente, de acuerdo al signo de la energía inicial $E(0)$.

Observemos que las estimativas $(4,15)$ y $(4,16)$ son equivalentes, es decir $\sqrt{a}=-J^{\prime}\left(t_{0}\right)$.

Desde que $\lim _{t \rightarrow T_{*}^{-}} J(t)=0$, se tiene

$$
\lim _{t \rightarrow T_{*}^{-}} A(t)=\infty
$$

De aquí y la desigualdad de Sobolev-Poincaré, se deduce

$$
\lim _{t \rightarrow T_{*}^{-}}\left[\|u(t)\|^{2}+\|v(t)\|^{2}\right]=\infty .
$$

Con todo esto se concluye la demostración del Teorema 4.8.

Observación 4.9. La elección de la constante positiva $T_{1}$ en $(4,12)$ se consige con algunas consideraciones. Las discusiones son similares como en [14]. Omitimos los detalles.

\section{Agradecimiento}

Al Consejo Superior de Investigación del Vicerrectorado de Investigación de la UNMSM, por el apoyo financiero otorgado para la ejecución del Proyecto de Estudio de Investigación 2012 con código: 121401121, cuyo primer resultado es la presente publicación. 


\section{REFERENCIAS BIBLIOGRÁFICAS}

[1] Brézis. H.. Análisis funcional, Alianza Editorial, Madrid, 1984.

[2] Castro, N. N. O., A nonlinear evolution system of partial differential equations with pLaplacian and negative nonlineariry, Proceedings of 10th WSEAS International Conference on APLLIED MATHEMATICS, Dallas, Texas, USA, November 1-3, 2006.

[3] Chen, C., Yao, H., and Shao, L., Global existence, uniqueness, and asymptotic behavior of solution for p-Laplacian type wave equation, Journal of Inequalities and Applications Volume 2010, Article ID 216760, 15 pages.

[4] Coddington E. A. and Levinson N., Theory of ordinary differential equations, McGraw-Hill, New York, 1955.

[5] GaoGao, H., Ma, T. F., Global solutions for a nonlinear wave equation with the pLaplacian operator, EJQTDE, 1999, No. 11.

[6] Li, M.-R., Tsai L.-Y., Existence and nonexistence of global solutions of some system of semilinear wave equations, Nonlinear Analysis 54 (2003) 1397-1415.

[7] Lima, O. A., Lourêdo, A. T., Marinho, A. O., Weak solutions for a strongly-coupled nonlinear system, Electronic Journal of Differential Equations, Vol. 2006 (2006), No. 130, pp. 1-18.

[8] Ma, T. F., Soriano, J. A., On weak solutions for an evolution equation with exponential nonlinearities, Nonlinear Analysis 37 (1999) 1029-1038.

[9] Milla Miranda, M. and Medeiros, L. A., On the existence of global solutions of a coupled nonlinear Klein-Gordon equations, Funkcialaj Ekvaciaj, 30 (1987) 147-161.

[10] Nishihara, K., On a global solution of some quasilinear hyperbolic equation, Tokyo J. Math Vol. 7, No. 2, 437-459, 1984.

[11] Quispe Méndez, T., Singularidad de soluciones para una ecuacón de onda degenerada no lineal con término disipativo,PESQUIMAT Revista de la Fac. CC. MM. de la Universidad Nacional Mayor de San Marcos Vol. XI, No.1, pp 41-54, LIMA-PERÚ. Octubre 2008.

|12| Quispe Méndez, T., Solución local para una ecuación del calor degenerada no lineal, PESQUIMAT Revista de la Fac. CC. MM.de la Universidad Nacional Mayor de San Marcos, Vol. XI, No. 2, pp 56-70. LIMA-PERÚ, Octubre 2008

[13] Quispe Méndez, T. y Carrillo Díaz, L. E., Solución local de un sistema de Kirchhoff no lineal viscoelástico con término disipativo, PESQUIMAT, Revista de la F. C. M. de la Universidad Nacional Mayor de San Marcos Vol. XIII No.2, pp 40-58, Lima-Perú, Diciembre 2010.

[14] Quispe Méndez, T., Singularidad de soluciones para un sistema de Kirchhoff no lineal viscoelástico con término disipativo, PESQUIMAT, Revista de la F. C. M. de la Universidad Nacional Mayor de San Marcos Vol. XIV No.1, pp 46-57, Lima-Perú, Julio 2011.

[15] Segal, I., Nonlinear partial differential equations in quantum fiels theory, Proc. Symp. Appl. Math. A.M.S., 17, 210-226 (1965). 
[16] Showalter. R. E., Monotone operators in Banach space and nonlinear partial diffential equations, American Mathematical Society, 1997.

[17] Wu, S.-T. and Tsai, L.-Y., On a system of nonlinear wave equations of Kirchhoff type with a strong dissipation, Tamkang Journal of Mathematics Volume 38, Number 1, 1-20, Spring 2007.

[18] Yang, Z and Chen, G., Global existence of solutions for quasi-linear wave equations with viscous damping. Journal Mathematical Analysis and Applications 285 (2003) 604-618.

[19] Ye, Y., Exponential decay of energy for some nonlinear hyperbolic equations with strong dissipation, Advances in Difference Equations Volume 2010, Article ID 357404, 12 pages. 